\title{
Distribuição do cádmio nas frações químicas de um CAMBISSOLO FLÚVICO Alumínico gleissólico
}

\author{
Cadmium distribution in chemical fractions of a Fluventic Dystrudept soil \\ Eduardo da Silva Daniel*, David José Miquelluti, Mari Lucia Campos, Marta de Moura Madeira \&
Dreyce Kisholli Bueno
}

Universidade do Estado de Santa Catarina, Lages, SC, Brasil. *Autor para correspondência: edudaniel@hotmail.com.

Submissão:12/01/2017 | Aceite: 12/06/2018

\begin{abstract}
RESUMO
A compreensão da distribuição dos elementos-traço às frações químicas do solo, mobilidade e concentração total são imprescindíveis no diagnóstico da contaminação e definição de estratégias de remediação de áreas contaminadas. Este trabalho teve como objetivo observar o comportamento do $\mathrm{Cd}$ nas diferentes frações químicas e do $\mathrm{Cd}$ total através da extração por água régia na camada superficial de um CAMBISSOLO FLÚVICO Alumínico gleisólico. Após a contaminação com doses crescentes e incubação do metal no solo, amostras foram analisadas através do protocolo de GOMES et al. (1997) para a extração sequencial, e através do protocolo ISO 11047 (1998) (água régia) para a extração total. A concentração de $\mathrm{Cd}$ foi determinada por espectrometria de absorção atômica. Os resultados foram submetidos à análise de variância, sendo ajustadas equações de regressão entre as variáveis analisadas e as doses de $\mathrm{Cd}$ aplicadas no solo e calculadas as proporções do $\mathrm{Cd}$ alocado em cada fração e do $\mathrm{Cd}$ total recuperado. $\mathrm{O}$ aumento das doses de $\mathrm{Cd}$ aplicadas no solo experimentado elevou a concentração total, e a concentração as suas frações químicas, em ordem crescente de intensidade: óxidos de ferro < solúvel < residual < matéria orgânica < trocável. Proporcionalmente, sob baixas concentrações, o Cd tem maior tendência de ligar-se à matéria orgânica, óxidos de ferro e à fração residual. Sob altas concentrações, as frações solúvel e trocável somadas, que são as que apresentam maior mobilidade no solo, são mais representativas que as demais. A extração do Cd total através do método da água régia retornou valores acima de $77 \%$ das doses aplicadas no solo, apresentando elevada eficiência para concentrações de até $200 \mathrm{mg} \mathrm{kg}^{-1}$.
\end{abstract}

PALAVRAS-CHAVE: elementos-traço, extração sequencial, poluição de solos.

\begin{abstract}
Understanding of trace elements distribution in different soil chemical fractions and of the total mobility and concentration is essential for the diagnosis of contamination and the definition of remediation strategies for contaminated sites. This study aims to evaluate $\mathrm{Cd}$ behavior in the soil chemical fractions and $\mathrm{Cd}$ total concentration extracted by aqua regia of a Fluventic Dystrudept. After the contamination with increasing doses applied and incubation of the metal in the soil, samples were analyzed following the protocol described by GOMES et al. (1997) for sequential fractionation, and the ISO 11047 (1998) (aqua regia) for total extraction. $\mathrm{Cd}$ determination was performed by atomic absorption spectrometry. The results were subjected to analysis of variance. Regression equations were adjusted between the variables analyzed and the Cd levels were applied to the soil. Subsequently, the proportions of the Cd allocated in each fraction and the total recovered $\mathrm{Cd}$ were calculated. The increase in $\mathrm{Cd}$ levels applied to the soil of the experiment increased total $\mathrm{Cd}$ concentration and the concentration in the chemical fractions in the following order of intensity: iron oxides < soluble < residual < organic matter < exchangeable. Proportionally, under low concentrations, the element has higher tendency to bind with organic matter, iron oxides and residual fraction than with soluble and exchangeable fractions combined. In high concentrations, a combination of soluble and exchangeable fractions, which are the most mobile fractions in the soil, is more representative than other fractions. The extraction of total $\mathrm{Cd}$ by the aqua regia method returned values above $77 \%$ of the applied doses in the soil, presenting high efficiency for concentrations up to $200 \mathrm{mg} \mathrm{kg}^{-1}$.
\end{abstract}

KEYWORDS: trace elements, sequential extraction, soil pollution. 


\section{INTRODUÇÃO}

Níveis de $\mathrm{Cd}$ acima dos teores naturais na camada superficial dos solos refletem o impacto antropogênico. Nessa camada, interações instáveis podem provocar a contaminação da água, da subsuperfície e do lençol freático, aumentando o potencial de poluição do metal, sobretudo naqueles solos com alto fluxo hídrico (VANĚK et al. 2005).

A intoxicação por Cd ocorre principalmente pela ingestão de tecidos de plantas acumuladoras que crescem em meios contaminados, podendo acarretar em doenças crônicas e distúrbios fisiológicos em organismos animais NORDBERG 2009, (McLAUGHLIN et al. 2011).

A habilidade de absorção pela planta, espécies químicas e mobilidade no solo são fatores que governam a fitodisponibilidade dos elementos. Por sua vez, a mobilidade no solo depende da força de ligação entre o metal e a fração química em que se encontra. Os principais fatores que influenciam a força de ligação do $\mathrm{Cd}$ ao solo são $\mathrm{o} \mathrm{pH}$, a força iônica, a qualidade e teores de matéria orgânica e de constituintes inorgânicos, e a concentração de outros elementos-traço catiônicos (LOGANATHAN et al. 2012).

Grande parte dos métodos de extração consideram o elemento isolado, associado a uma fração específica. Este tipo de análise baseia-se, geralmente, no uso de uma única solução extratora, sendo então designada de extração única. Porém, em estudos de poluição ambiental, interessa, muitas vezes, avaliar simultaneamente a disponibilidade ambiental de determinado poluente no solo a curto e longo prazo, possibilitando avaliar o destino destes poluentes no ambiente e/ou definir estratégias de remediação adequadas (RAO et al. 2008). Para isso, é necessário quantificar o elemento nas diferentes partições químicas do solo.

De acordo com VIOLANTE et al. (2010), os elementos-traço no solo estão associados principalmente à solução, superfícies inorgânicas, matéria orgânica, minerais primários e secundários e óxidos. Metodologias de análise baseadas no fracionamento químico desses elementos têm sido buscadas ao longo do tempo, levando-se em consideração características intrínsecas de cada solo (RAO et al. 2008).

Frações carbonatadas de solos alcalinos têm sido consideradas na extração sequencial de elementos-traço (HASS \& FINE 2010). No entanto, em solos ácidos de regiões tropicais, as frações ligadas aos óxidos de ferro e alumínio possuem representatividade muito superior àquela (GOMES et al. 1997). Metodologias de extração sequencial desses elementos têm sido adaptadas para solos tropicais. Dentre elas, o método proposto por GOMES et al. (1997), além de desconsiderar a fração ligada a carbonatos, inclui a fração ligada a óxidos de alumínio, consideravelmente presente em solos muito intemperizados.

A quantidade total do elemento no solo também influencia a força de ligação entre o metal e a fração química em que se encontra. Para a determinação da concentração total, digestões empregando ácido fluorídrico (HF) para quebra dos compostos silicatados são necessárias, no entanto, são extremamente complexas e perigosas. A extração através de água régia possui eficiência tão alta quanto aqueles métodos que utilizam HF (89 a 110\%) (SASTRE 2002), e é alternativa mais simples e segura. Considerando que a Resolução do CONAMA no 420 de 2009 (BRASIL 2009) reconhece a legalidade do uso de métodos da Organização Internacional de Normatização (ISO), o método de extração por água régia ISO 11047 (1998) continua sendo um dos mais utilizados na abertura de amostras de solo.

Os solos aluviais representam um grupo específico de solo que pode ser contaminado por elementostraço não só pela deposição direta, mas também pela água contaminada durante inundações, as quais ampliam a capacidade de contaminação das fontes poluidoras (VANĚK et al. 2005).

O aumento da exposição do solo ao Cd nesse tipo de solo pode resultar em maiores proporções do metal alocado nas frações mais móveis, incorrendo em riscos elevados de contaminação ao meio ambiente e organismos.

Baseado nesses preceitos, este trabalho teve como objetivo determinar a resposta da partição do elemento entre as diferentes frações químicas e do teor de $\mathrm{Cd}$ total obtido através do método da água régia, em relação às doses de $\mathrm{Cd}$ aplicadas em amostras da camada superficial de um CAMBISSOLO FLÚVICO Alumínico gleissólico.

\section{MATERIAL E MÉTODOS}

O presente estudo foi conduzido entre os meses de março e maio de 2013. O substrato constituiu-se da camada de $0-20 \mathrm{~cm}$ de um solo coletado em ambiente de campo nativo, no município de Bocaina do Sul, $\mathrm{SC}$, em locais contíguos a leitos de corpos d'água, os quais encontram-se permanentemente ou periodicamente inundados. De acordo com a caracterização desse ponto, o solo é classificado taxonomicamente como CAMBISSOLO FLÚVICO Alumínico gleissólico (SANTOS 2013), de classe textural 
franco-argilo-siltosa. As características do solo são apresentadas na Tabela 1.

Tabela 1. Características químicas e físicas da camada superficial $(0-20 \mathrm{~cm})$ do CAMBISSOLO FLÚVICO Alumínico gleissólico coletado no município de Bocaina do Sul, SC, utilizado no experimento.

Table 1. Chemical and physical characteristics of the topsoil $(0-20 \mathrm{~cm})$ of the Fluventic Dystrudept collected in the city of Bocaina do Sul, SC.

\begin{tabular}{|c|c|c|}
\hline Característica & Unidade & Valor \\
\hline $\mathrm{pH}$ em água ${ }^{(1)}$ & & 5,00 \\
\hline $\mathrm{pH}$ em $\mathrm{CaCl}_{2}(1)$ & & 3,70 \\
\hline$\left.A\right|^{3+(2)}$ & $\mathrm{cmol}_{c} \mathrm{dm}^{-3}$ & 2,20 \\
\hline $\mathrm{Ca}^{2+(2)}$ & $\mathrm{cmol}_{c} \mathrm{dm}^{-3}$ & 5,90 \\
\hline $\mathrm{Mg}^{2+(2)}$ & $\mathrm{cmol}_{\mathrm{cdm}}{ }^{-3}$ & 1,20 \\
\hline $\mathrm{K}^{(2)}$ & $\mathrm{mg} \mathrm{kg}^{-1}$ & 49,00 \\
\hline $\mathrm{Na}{ }^{(2)}$ & $\mathrm{mg} \mathrm{kg}^{-1}$ & 39,00 \\
\hline$P^{(2)}$ & $\mathrm{mg} \mathrm{kg}^{-1}$ & 4,20 \\
\hline $\mathrm{H}+\mathrm{Al}(2)$ & $\mathrm{cmol}_{\mathrm{cdm}} \mathrm{C}^{3}$ & 5,07 \\
\hline Soma de bases & $\mathrm{cmol}_{\mathrm{c}} \mathrm{dm}^{-3}$ & 7,23 \\
\hline СTC efetiva & $\mathrm{cmol}_{\mathrm{c}} \mathrm{dm}^{-3}$ & 9,43 \\
\hline CTC em pH $7^{(2)}$ & $\mathrm{cmol}_{\mathrm{c}} \mathrm{dm}^{-3}$ & 15,96 \\
\hline Saturação de bases & $\%$ & 45,27 \\
\hline Saturação de Al & $\%$ & 23,34 \\
\hline Matéria Orgânica (2) & $\%$ & 9,52 \\
\hline $\operatorname{Areia}^{(1)}$ & $\mathrm{g} \mathrm{kg}^{-1}$ & 127,00 \\
\hline Silte ${ }^{(1)}$ & $\mathrm{g} \mathrm{kg}^{-1}$ & 525,00 \\
\hline Argila ${ }^{(1)}$ & $\mathrm{g} \mathrm{kg}^{-1}$ & 348,00 \\
\hline $\mathrm{Fe}_{2} \mathrm{O}_{3}{ }^{(3)}$ & $\mathrm{g} \mathrm{kg}^{-1}$ & 72,92 \\
\hline $\mathrm{Al}_{2} \mathrm{O}_{3}(3)$ & $\mathrm{g} \mathrm{kg}^{-1}$ & 175,64 \\
\hline
\end{tabular}

${ }^{(1)}$ Determinação de acordo com DONAGEMA (2011); ( ${ }^{2}$ Determinação de acordo com TEDESCO et al. (1995); ${ }^{(3)}$ Extração por ataque sulfúrico e determinação por espectrofotometria de absorção atômica, de acordo com DONAGEMA (2011).

Após a coleta, o solo foi destorroado, homogeneizado e quarteado manualmente com auxílio de pá quadrada e quarteador, seco ao ar e tamisado em peneira de $2 \mathrm{~mm}$ de abertura de malha. Em seguida, 0 mesmo foi disposto em vasos plásticos, em quantidade de $5,0 \mathrm{~kg}$ cada, constituindo, desta maneira, as unidades experimentais.

Subsequentemente, o solo contido nos vasos foram contaminado com Cd utilizando-se soluções de nitrato de cádmio $\left(\mathrm{Cd}\left(\mathrm{NO}_{3}\right)_{2}\right)$ que resultaram em doses equivalentes a $0 ; 10 ; 50 ; 100$ e $200 \mathrm{mg} \mathrm{kg}^{-1}$. Essas doses corresponderam aos tratamentos, com quatro repetições cada, arranjados de acordo com um delineamento inteiramente casualizado.

As doses adicionadas foram definidas de acordo com os valores orientadores de substâncias tóxicas para a qualidade dos solos indicados pela Resolução do CONAMA no 420 de 2009 (BRASIL 2009) e com testes prévios de adsorção.

Em seguida, o solo foi incubado por 45 dias em casa de vegetação, para a estabilização das condições químicas. A umidade do solo, nesse ínterim, foi mantida em $80 \%$ da capacidade de retenção de água, através do acréscimo periódico de água deionizada.

Transcorrido o período de incubação, o solo de cada unidade experimental foi seco ao ar, destorroado, tamisado em peneira de $2 \mathrm{~mm}$ de abertura de malha e homogeneizado. $\mathrm{O}$ quarteamento seguiu-se até serem obtidas amostras para análise de aproximadamente $10 \mathrm{~g}$, as quais foram subsequentemente secas em estufa de ventilação forçada à $65^{\circ} \mathrm{C}$ durante $24 \mathrm{~h}$.

Em relação às análises, primeiramente foi realizada a extração sequencial do elemento nas frações químicas do solo por meio do método proposto por GOMES et al. (1997). Este esquema de extração divide as frações químicas do solo em solúvel, trocável, ligado à matéria orgânica, aos óxidos de ferro, aos óxidos de alumínio e residual.

Considerando-se este método, algumas modificações se fizeram necessárias: (a) quantificação com 
base na massa em vez de volume, para facilitar a comparação dos resultados; (b) separação dos extratos da amostra através de pipetagem do sobrenadante, em vez da utilização de papel filtro; (c) transferência do resíduo da extração da fração ligada aos óxidos de ferro para tubos de digestão, no lugar de cadinhos de teflon, seguido de secagem do conteúdo em estufa, para posterior digestão da fração residual; (d) utilização do método ISO 11047 (1998) para a digestão da fração residual, substituindo a combinação dos ácido nítrico, perclórico e fluorídrico.

A centrifugação das amostras ocorreu a 3.500 rpm, durante 20 min. Após cada extração, adicionou-se $5 \mathrm{~mL}$ de água deionizada ao resíduo de solo, sendo a amostra novamente centrifugada, e o sobrenadante gerado descartado, para que toda a solução extratora da fração anterior fosse eliminada. A seguir, foi realizada a abertura das amostras, com o objetivo de quantificar o $\mathrm{Cd}$ total no solo, através do método ISO 11047 (1998) (água régia).

As determinações de $\mathrm{Cd}$ foram realizadas por espectrofotômetro de absorção atômica de alta resolução com atomização em chama ar-acetileno ContrAA-700 (Analytyc Jena, Alemanha). As curvas analíticas foram construídas utilizando-se as soluções extratoras como solventes, suprimindo-se, desta maneira, o efeito matriz nas leituras. Os pontos das curvas foram definidos em $0 ; 0,5 ; 1,0 ; 1,5$ e $2,0 \mathrm{mg} \mathrm{L}^{-1}$ de Cd.

Foram utilizadas oito provas em branco para a determinação dos limites de detecção do instrumento (LDI), os quais corresponderam a $0,0049 \mathrm{mg} \mathrm{L}^{-1}$ para a fração solúvel; $0,0101 \mathrm{mg} \mathrm{L}^{-1}$ para a fração trocável; $0,0051 \mathrm{mg} \mathrm{L}^{-1}$ para a fração ligada à matéria orgânica; $0,0087 \mathrm{mg} \mathrm{L}^{-1}$ para a fração ligada aos óxidos de alumínio; $0,0061 \mathrm{mg} \mathrm{L}^{-1}$ para a fração ligada aos óxidos de ferro; $0,0058 \mathrm{mg} \mathrm{L}^{-1}$ para a fração residual e Cd total no solo extraído por água régia. Todas as análises químicas para as determinações do Cd foram realizadas em tréplica.

A confiabilidade do método analítico empregado para a determinação do Cd total no solo foi avaliada utilizando-se a amostra de solo certificada pelo National Institute of Standards and Technology (NIST), SRM 2709a (San Joaquin), com acurácia na recuperação de $100 \pm 10 \%$.

Os dados obtidos foram testados quanto à normalidade por meio do teste de Shapiro-Wilk e quanto à homogeneidade das variâncias por meio do teste de Bartlett.

A análise do comportamento da concentração de Cd nas frações químicas e total (y) em função das doses de $\mathrm{Cd}$ aplicadas no solo $(x)$ foi realizada através do ajuste do modelo linear apresentado na Equação 1:

$$
y=a+b x
$$

Equação 1

Foram então calculados os percentuais de recuperação do Cd aplicado ao solo, e de extração do Cd nas frações em relação ao $\mathrm{Cd}$ total do solo (água régia). Também foram calculados os índices de mobilidade do metal, considerando as frações solúvel e trocável como altamente lábeis. Esses índices foram expressos através do fator de mobilidade (FM), baseado na Equação 2 (KABALA \& SINGH 2006):

$$
\mathrm{FM}=\left[\frac{\mathrm{Cd} \text { solúv } \theta \text { l }+\mathrm{Cd} \text { trocável }}{\text { Cd total (água régi a) }}\right] \times 100(\%)
$$

Equação 2

Por fim, a concentração de Cd total obtido através do somatório das concentrações de Cd nas frações químicas e através da extração por água régia foram comparadas em cada dose aplicada utilizando-se 0 teste $t$ de Student (amostras pareadas). Todas as análises estatísticas consideraram $p<0,05$.

\section{RESULTADOS}

Foi observado um aumento linear da concentração de $\mathrm{Cd}$ em todas as frações químicas do solo de acordo com o acréscimo da dose do elemento adicionado (Figura $1 \mathrm{~A}$ ), à exceção da fração óxidos de alumínio. Este aumento foi mais pronunciado na fração trocável do solo, seguida da fração ligada à M.O., residual, solúvel e ligada aos óxidos de ferro.

$\mathrm{Na}$ fração solúvel, as concentrações obtidas representaram 7,2; 7,3; 7,5;7,9 e 8,8\% e, na fração trocável, corresponderam a 30,1; 40,8; 39,6; 45,7 e 49,3\% em relação ao Cd total no solo, respectivamente, para as doses aplicadas de 0; 10; 50; 100 e $200 \mathrm{mg} \mathrm{kg}^{-1}$ (Tabela 2).

$\mathrm{Na}$ fração ligada à matéria orgânica, as concentrações extraídas corresponderam a 21,7; 17,9; 15,0; 14,1 e 13,4\%; na fração ligada aos óxidos de ferro, corresponderam a 50,6;10,7;4,2;3,4 e 3,9\% e na fração residual, 42,2; 25,5; 14,7; 12,0; e 11,6\%, em relação ao Cd total do solo, para as respectivas doses aplicadas de $0 ; 10 ; 50 ; 100$ e $200 \mathrm{mg} \mathrm{kg}^{-1}$, enquanto que os teores de Cd ligado aos óxidos de alumínio 
ficaram abaixo do limite de detecção do instrumento de leitura (Tabela 2).

A.

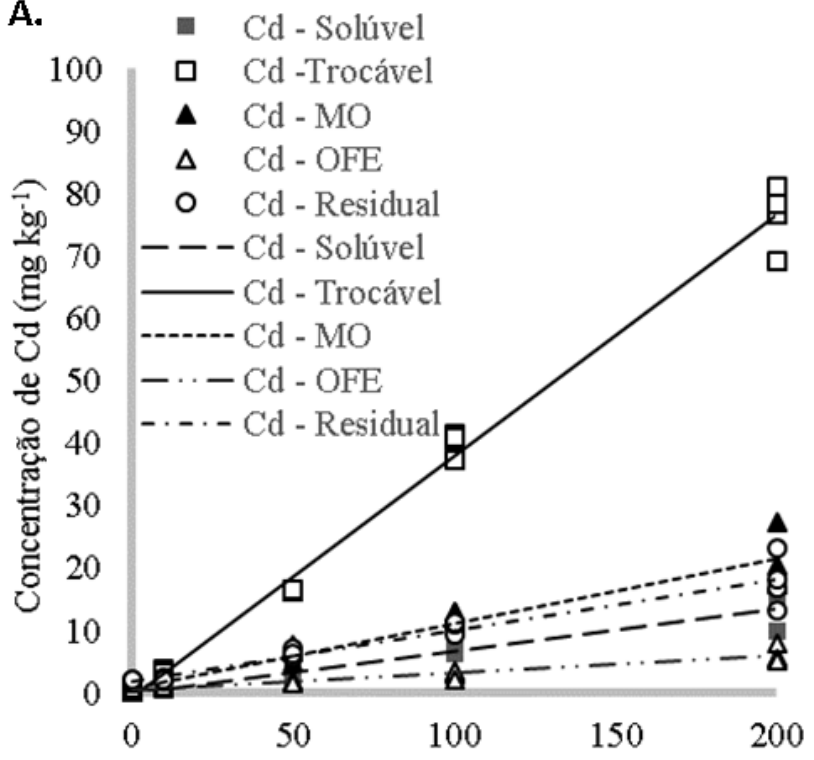

B.

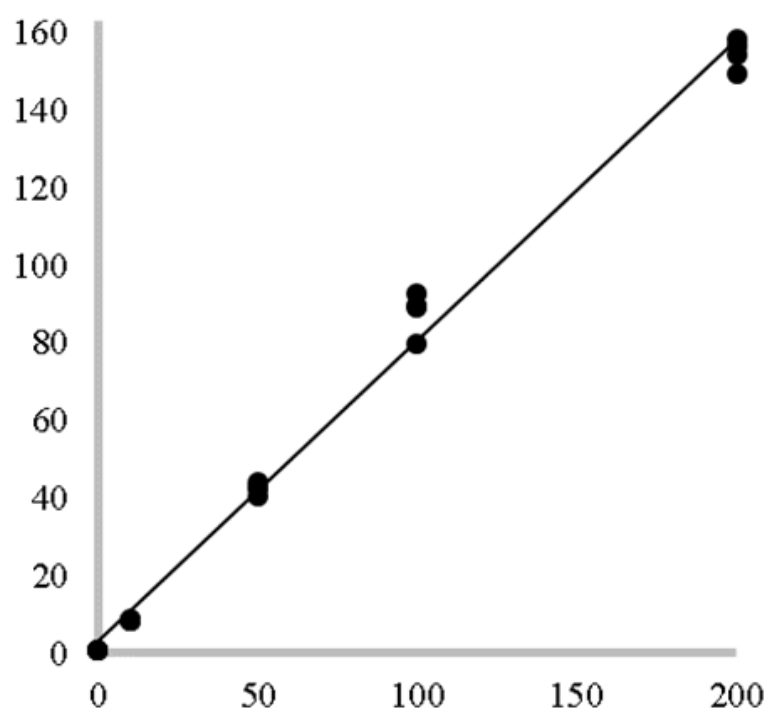

Dose de Cd aplicada no solo $\left(\mathrm{mg} \mathrm{kg}^{-1}\right)$

Figura 1. Cd associado às diferentes frações químicas $(A)$ e $C d$ total $(B)$ da camada superficial $(0-20 \mathrm{~cm})$ do CAMBISSOLO FLÚVICO Alumínico gleissólico coletado no município de Bocaina do Sul, SC, em função de doses crescentes do metal.

Figure 1. Cd associated with the different chemical fractions $(A)$ and total $C d(B)$ of the topsoil $(0-20 \mathrm{~cm})$ of the Fluventic Dystrudept collected in the city of Bocaina do Sul, SC according to increasing doses of the metal.

A concentração de $\mathrm{Cd}$ total obtido através da extração por água régia também aumentou linearmente à medida que se elevou as doses de $\mathrm{Cd}$ aplicadas no solo (Figura 1B). As proporções recuperadas perfizeram 85,3; 84,9; 87,7 e 77,3\% das doses aplicadas de 10; 50; 100 e $200 \mathrm{mg} \mathrm{kg}^{-1}$, respectivamente. Já o fator de mobilidade para o Cd no solo correspondeu a 37,$3 ; 48,1 ; 47,1 ; 53,6$ e 58,1\%, para as doses aplicadas de $0 ; 10 ; 50 ; 100$ e $200 \mathrm{mg} \mathrm{kg}^{-1}$, respectivamente (Tabela 2).

Tabela 2. Concentração de Cd nas frações solúvel (SOL), trocável (TRO), matéria orgânica (MO), ligada aos óxidos de alumínio (OAL), aos óxidos de ferro (OFE), residual (RES) e Cd total (TOT) da camada superficial $(0-20 \mathrm{~cm})$ do CAMBISSOLO FLÚVICO Alumínico gleissólico coletado no município de Bocaina do Sul, SC em função das doses crescentes do metal.

Table 2. Cd concentration in the soluble (SOL), exchangeable (EXC), organic matter (OM), bound to aluminum oxides (ALO), to iron oxides (IO) and residual (RES) fractions and total Cd (TOT) in the topsoil $(0-20 \mathrm{~cm})$ of the Fluventic Dystrudept collected in the city of Bocaina do Sul, SC, according to increasing doses of the metal.

\begin{tabular}{|c|c|c|c|c|c|c|c|}
\hline Dose & SOL & TRO & MO & OAL & OFE & RES & TOT \\
\hline \multicolumn{8}{|c|}{ 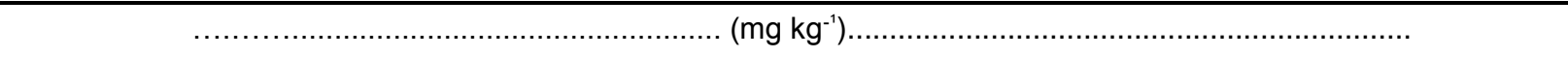 } \\
\hline 0 & $0,06 \pm 0,02^{(1)}$ & $0,25 \pm 0,03$ & $0,18 \pm 0,02$ & $<\mathrm{LD}^{(2)}$ & $0,42 \pm 0,05$ & $0,35 \pm 0,04$ & $0,83 \pm 0,09$ \\
\hline 10 & $0,62 \pm 0,07$ & $3,48 \pm 0,31$ & $1,53 \pm 0,18$ & $<\mathrm{LD}$ & $0,91 \pm 0,10$ & $2,18 \pm 0,33$ & $8,53 \pm 0,96$ \\
\hline 50 & $3,19 \pm 0,52$ & $16,80 \pm 0,93$ & $6,38 \pm 0,48$ & $<\mathrm{LD}$ & $1,78 \pm 0,21$ & $6,26 \pm 0,47$ & $42,45 \pm 2,52$ \\
\hline 100 & $6,93 \pm 0,89$ & $40,10 \pm 2,46$ & $12,37 \pm 0,80$ & $<\mathrm{LD}$ & $2,96 \pm 0,39$ & $10,50 \pm 1,04$ & $87,73 \pm 5,87$ \\
\hline 200 & $13,58 \pm 1,41$ & $76,26 \pm 7,91$ & $20,71 \pm 1,68$ & $<L D$ & $6,11 \pm 0,45$ & $17,90 \pm 2,24$ & $154,63 \pm 11,11$ \\
\hline
\end{tabular}

${ }^{(1)}$ Média \pm desvio-padrão dos valores; $n=3$; (2)LD: Limite de detecção do instrumento.

Os coeficientes das equações de regressão lineares ajustadas entre os teores de Cd extraídos das frações e Cd total, e as doses do metal adicionadas ao solo, podem ser visualizados na Tabela 3. 
Tabela 3. Coeficientes das equações de regressão lineares ajustadas entre os teores de Cd nas frações químicas e $\mathrm{Cd}$ total e as doses do metal adicionadas à camada superficial $(0-20 \mathrm{~cm})$ do CAMBISSOLO FLÚVICO Alumínico gleissólico coletado no município de Bocaina do Sul, SC.

Table 3. Coefficients of the adjusted linear regression equations between the $\mathrm{Cd}$ in the chemical fractions, total $\mathrm{Cd}$, and the metal doses added to the topsoil $(0-20 \mathrm{~cm})$ of the Fluventic Dystrudept collected in the city of Bocaina do Sul, SC.

\begin{tabular}{lcccc}
\hline Fração química & $\mathrm{a}$ & $\mathrm{b}$ & Probabilidade & $\mathrm{R}^{2}$ \\
\hline Solúvel & $-0,04$ & 0,07 & $<0,01$ & 0,96 \\
Trocável & $-0,54$ & 0,37 & $<0,01$ & 0,99 \\
Matéria orgânica & 0,81 & 0,10 & $<0,01$ & 0,93 \\
Óxidos de ferro & 0,44 & 0,03 & $<0,01$ & 0,93 \\
Residual & 1,25 & 0,08 & $<0,01$ & 0,92 \\
Total & 2,90 & 0,78 & $<0,01$ & 0,99 \\
\hline
\end{tabular}

Os valores de $\mathrm{Cd}$ total obtidos via água régia foram menores que os valores resultantes do somatório das concentrações do metal nas frações químicas do solo para as doses de 0 e $10 \mathrm{mg} \mathrm{kg}^{-1}$, e maiores para as doses de 50 a $200 \mathrm{mg} \mathrm{kg}^{-1}$ (Tabela 4).

Tabela 4. Cd total extraído por água régia e Cd total resultante do somatório das concentrações do metal nas frações químicas da camada superficial $(0-20 \mathrm{~cm})$ do CAMBISSOLO FLÚVICO Alumínico gleissólico coletado no município de Bocaina do Sul, SC, em função das doses crescentes do metal.

Table 4. Total $\mathrm{Cd}$ extracted by aqua regia and total $\mathrm{Cd}$ resulting from the sum of the metal concentrations in the chemical fractions of the topsoil $(0-20 \mathrm{~cm})$ of the Fluventic Dystrudept collected in the city of Bocaina do Sul, SC, according to increasing doses of the metal.

\begin{tabular}{llcl}
\hline Dose aplicada & \multicolumn{1}{c}{ Cd total água régia } & Cd total nas frações & Erro- padrão \\
\hline & & $\ldots \ldots \ldots \ldots . . .\left(\mathrm{mg} \mathrm{kg}^{-1}\right) \ldots \ldots \ldots \ldots \ldots \ldots \ldots \ldots \ldots$ & \\
\hline 0 & 0,83 & $1,26^{*}$ & 0,04 \\
10 & 8,33 & $8,93^{*}$ & 0,08 \\
50 & 42,45 & $33,98^{*}$ & 0,90 \\
100 & 87,73 & $72,86^{*}$ & 1,57 \\
200 & 154,63 & $135,58^{*}$ & 4,23 \\
\hline
\end{tabular}

*diferença significativa $(p<0,05)$ pelo teste t de Student (amostras pareadas); $n=3$.

\section{DISCUSSÂO}

Considerando-se o aumento proporcional de Cd nas frações solúvel e trocável somadas (sobretudo devido à fração trocável), que são as mais prontamente disponíveis no solo, à medida que as doses se elevaram, constata-se que a disponibilidade do metal relativa ao $\mathrm{Cd}$ total é maior sob altas concentrações no solo, reduzindo-se para concentrações menores.

O Cd presente na solução do solo é altamente dependente do Cd trocável, o qual encontra-se, em grande parte, adsorvido eletrostaticamente às cargas negativas dos colóides (PIERANGELI et al. 2007). Dessa maneira, a capacidade de troca de cátions (CTC) afeta sobremaneira a disponibilidade do Cd no solo (LINHARES et al. 2009). ALLEONI et al. (2005) encontraram correlação positiva entre a adsorção máxima de Cd com a CTC total do solo. Os altos valores de Cd trocável para todas as doses aplicadas e o alto valor de CTC apresentados pelo solo avaliado corroboram tal informação.

Considerando-se a concentração de Cd nas frações solúvel e trocável conjuntamente, os resultados deste estudo concordam com GOMES et al. (1997) que, trabalhando com extração fracionada em LATOSSOLO VERMELHO-AMARELO Húmico, constataram maior distribuição do elemento nessas frações $(>50 \%)$ que nas demais. COSTA et al. (2007) também obtiveram altos teores de Cd disponível em uma extração sequencial do elemento em diferentes solos contaminados, os quais variaram de 18,2 a $51,1 \%$ do Cd total no solo.

De maneira contrária ao $\mathrm{Cd}$ na fração trocável, a proporção de Cd associado à matéria orgânica foi reduzida conforme o aumento da concentração total no solo, de forma que sob baixas concentrações, a 
complexação do metal a essa fração é um fenômeno mais representativo, comparativamente ao que ocorre em doses mais elevadas. Esses resultados reforçam a resposta obtida por HONMA et al. (2015), os quais concluíram que com o aumento proporcional do Cd ligado à fração trocável, há uma diminuição da proporção do Cd associado à matéria orgânica, tanto para solos contaminados quanto para solos não contaminados.

Mesmo com valores de matéria orgânica muito semelhantes $(<7,90 \%)$ ao teor constatado nesse estudo, GOMES et al. (1997) observaram menores proporções de Cd ligado a essa fração. No entanto, as taxas de $\mathrm{Cd}$ associadas à fração trocável naquele trabalho foram superiores ao deste. Em CAMBISSOLOS HÚMICOS com CTC e teores de matéria orgânica mais baixos, COSTA et al. (2007) encontraram o Cd associado a essa fração em proporções variando de 39,8 a $45,6 \%$, porém com valores de 20,3 a $25,5 \%$ na fração trocável.

Semelhantemente ao que ocorreu ao $\mathrm{Cd}$ associado à matéria orgânica, a proporção de $\mathrm{Cd}$ na fração óxidos de ferro em relação ao $\mathrm{Cd}$ total do solo diminuiu à medida em que houve o aumento das doses aplicadas no solo, tendo representatividade alta nas doses mais baixas, e baixa nas doses mais altas. HADLICH \& UCHA (2010) encontraram altas proporções de Cd ocluso em óxidos de ferro e manganês $(\geq 47 \%)$ em um solo com baixos teores de Cd total, mesmo com menores teores de ferro $\left(\leq 14,35 \mathrm{mg} \mathrm{kg}^{-1}\right)$. GOMES et al. (1997) observaram uma redução da proporção do Cd ligado aos óxidos de ferro com o aumento da concentração de Cd total no solo. Os autores desse trabalho encontraram valores de recuperação abaixo de $21,05 \%$ do $\mathrm{Cd}$ total nessa fração.

Os óxidos de ferro proporcionam substancial efeito na adsorção do $\mathrm{Cd}$ no solo por meio de ligações covalentes e específicas em sítios de alta afinidade. No entanto, os resultados indicam que após a saturação desses sítios, a concentração residual é elevada em relação aos outros cátions, e o metal é capaz de competir pelos sítios de baixa afinidade (ALLEONI et al. 2005), reduzindo a proporção associada a essa fração e aumentando aquela associada à fração trocável do solo.

Apesar dos óxidos de alumínio apresentarem concentração considerável no solo avaliado $(175,64 \mathrm{~g}$ $\mathrm{kg}^{-1}$ ), os resultados demonstraram uma quantidade de Cd ligada a essa fração menor que o limite de detecção do aparelho $(0,0087 \mathrm{mg} \mathrm{L})$. Este fato indica uma menor adsorção do Cd aos óxidos de alumínio que aos óxidos de ferro e às argilas silicatadas, como observado por GOMES et al. (1997). Não se descarta a possibilidade de baixa eficiência do extrator utilizado para o $\mathrm{Cd}$ associado à essa fração $(\mathrm{NaOH})$, tampouco que a ordem de extração das diferentes frações possa afetar a recuperação do metal nessa fração.

A proporção de $\mathrm{Cd}$ residual em relação ao $\mathrm{Cd}$ total apresentou comportamento similar à proporção de Cd associado à matéria orgânica e aos óxidos de ferro: reduziu com a elevação das doses aplicadas no solo, com altos valores nas doses mais baixas, indicando uma maior afinidade do elemento às argilas $\mathrm{e}$ outros óxidos do solo em baixas contaminações. KABALA \& SINGH (2006) observaram uma maior participação do $\mathrm{Cd}$ residual em solos não contaminados, em comparação aos contaminados. GOMES et al. (1997) também corroboram os resultados obtidos, com uma variação de 15,8 a $6,7 \%$ do Cd na fração residual em relação ao $\mathrm{Cd}$ total quando as doses aplicadas no solo foram aumentadas de 2,4 para 7,2 mg $\mathrm{kg}^{-1}$.

O Cd foi bastante retido nas frações orgânicas, óxidos de ferro e residuais, sobretudo nas doses mais baixas, indicando que em condições de baixa competição com outros cátions, tem tendência de manter ligações mais fortes com o solo, permanecendo complexado à matéria orgânica e adsorvido especificamente a óxidos de ferro e minerais silicatados. No entanto, o predomínio do Cd trocável sobre o $\mathrm{Cd}$ associado às demais frações a partir da dose de $10 \mathrm{mg} \mathrm{kg}^{-1}$ é motivo de preocupação quanto ao potencial de contaminação dos solos. Nessa fração, ele mantém ligações menos energéticas (eletrostáticas) e, sendo fracamente adsorvido, pode ser solubilizado e absorvido pelas plantas, adentrando na cadeia alimentar.

O aumento do fator de mobilidade conforme a elevação da concentração de $\mathrm{Cd}$ acentua o risco ambiental, uma vez que as camadas superficiais do solo estudado têm baixa capacidade de retenção do metal sob altas contaminações. Em estudo cujo objetivo foi examinar a mobilidade de elementos-traço através do perfil de solos tropicais contaminados, THOMASI et al. (2015) verificaram que o Cd teve a menor retenção e foi o mais susceptível à transferência do solo para outros compartimentos ambientais dentre vários metais avaliados.

Os solos aluviais, como o utilizado neste estudo, representam um grupo específico de solo que pode ser contaminado não só pela deposição direta de elementos-traço, mas também pela água contaminada durante inundações. Tal fato, aliado à alta mobilidade do $\mathrm{Cd}$, podem acentuar a transferência do metal no 
solo e aumentar o potencial de contaminação a partir das fontes do metal, conforme identificado por VANĚK et al. (2005).

Os valores da concentração de $\mathrm{Cd}$ total no solo obtidos através da extração por água régia concordam com os apresentados por SASTRE et al. (2002), e comprovam que esse método extrai a quase totalidade dos elementos-traço no solo, uma vez que a solução extratora só não alcança os metais que fazem parte da estrutura dos minerais silicatados.

Os maiores valores de $\mathrm{Cd}$ total obtidos pelo somatório das concentrações nas frações químicas do solo, para as doses de 0 e $10 \mathrm{mg} \mathrm{kg}^{-1}$, foram devidos à maior especificidade dos extratores utilizados, em relação ao método de extração de $\mathrm{Cd}$ total por água régia. Para as doses de 50 a $200 \mathrm{mg} \mathrm{kg}^{-1}$, tais extratores foram menos eficientes, uma vez que os maiores valores foram observados para o $\mathrm{Cd}$ total. Em ambos os casos, a diferença entre os valores foi significativa $(p<0,05)$. Resultados semelhantes foram encontrados por COSTA et al. (2007). Apesar das diferenças apontadas, existe uma forte correlação $(r=0,99)$ entre o somatório das concentrações de $\mathrm{Cd}$ nas frações químicas e aquelas obtidas para o Cd total no solo extraído por água régia.

A utilização de água régia como extrator do $\mathrm{Cd}$ ligado à fração residual pode explicar parte considerável das diferenças entre o somatório das concentrações de Cd nas frações e a dose aplicada, de maneira análoga ao que acontece na extração do $\mathrm{Cd}$ total do solo, já que essa solução não extrai os metais presentes nos silicatos (KELLER \& VÉDY 1994).

\section{CONCLUSÃO}

À medida que se aumenta a dose de $\mathrm{Cd}$, eleva-se a sua concentração nas frações químicas do CAMBISSOLO FLÚVICO Alumínico gleissólico, em ordem crescente de intensidade: óxidos de $\mathrm{Fe}<$ solúvel $<$ residual < matéria orgânica < trocável.

Em condições de baixa concentração no solo, o $\mathrm{Cd}$ tem a tendência de se associar às frações menos disponíveis, como a matéria orgânica, óxidos de ferro e residual. À medida que a concentração aumenta, as frações mais disponíveis como a trocável e a solução do solo são acionadas em maiores proporções. Em ambos os casos, há um alto potencial de mobilidade do metal no solo.

A extração do $\mathrm{Cd}$ total através do método da água régia recupera mais de $77 \%$ do $\mathrm{Cd}$ do solo, apresentando alta eficiência para concentrações até $200 \mathrm{mg} \mathrm{kg}^{-1}$.

\section{AGRADECIMENTOS}

Os autores agradecem à Coordenação de Aperfeiçoamento de Pessoal de Nível Superior (CAPES) e à Universidade do Estado de Santa Catarina (UDESC) pelo apoio financeiro.

\section{REFERÊNCIAS}

ALLEONI LRF et al. 2005. Atributos do solo relacionados à adsorção de cádmio e cobre em solos tropicais. Acta Scientiarum Agronomy 27: 729-737.

BRASIL. 2009. Ministério do Meio Ambiente. Conselho Nacional do Meio Ambiente. Resolução no 420 de 2009.

COSTA CN et al. 2007. Fracionamento sequencial de cádmio e chumbo em solos. Ciência Rural 37: 1323-1328.

DONAGEMA GK et al. 2011. Manual de Métodos de Análise de Solo. 2.ed. Rio de Janeiro: EMBRAPA. 226p.

GOMES PC et al. 1997. Extração fracionada de metais pesados em latossolo vermelho-amarelo. Revista Brasileira de Ciência do Solo 21: 543-551.

HADLICH GM \& UCHA JM. 2010. Distribution of cadmium in a cultivated soil in Britanny, France. Scientia Agricola 67: 731-736.

HASS A \& FINE P. 2010. Sequential selective extraction procedures for the study of heavy metals in soils, sediments, and waste materials - a critical review. Critical Reviews in Environmental Science and Technology 40: 365-399.

HONMA T et al. 2015. Relationship between cadmium fractions obtained by sequential extraction of soil and the soil properties in contaminated and uncontaminated paddy soils. Journal of Chemistry. 9p.

ISO 11047. 1998. Soil quality - Determination of cadmium, chromium, cobalt, copper, lead, manganese, nickel and zinc in aqua regia extracts of soil - Flame and electrothermal atomic absorption spectrometric methods. Washington: American National Standards Institute. 24p.

KABALA C \& SINGH BR. 2006. Distribution and forms of cadmium in soils near a copper smelter. Polish Journal of Environmental Studies 15: 90-97.

KELLER C \& VÉDY JC. 1994. Distribution of copper and cadmium fractions in two forest soils. Journal of Environmental Quality Abstract 23: 987-999.

LINHARES LA et al. 2009. Adsorção de cádmio e chumbo em solos tropicais altamente intemperizados. Pesquisa Agropecuária Brasileira 44: 291-299.

LOGANATHAN P et al. 2012. Cadmium sorption and desorption in soils: a review. Critical Reviews in Environmental 
Science and Technology 42: 489-533.

McLAUGHLIN MJ et al. 2011. Uptake of metals from soil into vegetables. In: SWARTJES FA (ed.). Dealing with contaminated sites: from theory towards practical application. Holanda: Springer. p. 325-367.

NORDBERG GF. 2009. Historical perspectives on cadmium toxicology. Toxicology and Applied Pharmacology 238: $192-$ 200.

PIERANGELI MAP et al. 2007. Comportamento sortivo, individual e competitivo, de metais pesados em Latossolos com mineralogia contrastante. Revista Brasileira de Ciência do Solo 31: 819-826.

RAO CRM et al. 2008. A review of the different methods applied in environmental geochemistry for single and sequential extraction of trace elements in soils and related materials. Water Air Soil Pollution 189: 291-333.

SANTOS HG et al 2013. Sistema Brasileiro de Classificação de Solos. 3.ed. Brasília: EMBRAPA. 353p.

SASTRE $\mathrm{J}$ et al. 2002. Determination of $\mathrm{Cd}, \mathrm{Cu}, \mathrm{Pb}$ and $\mathrm{Zn}$ in environmental samples: microwave-assisted total digested versus aqua regia and nitric acid extraction. Analytica Chimica Acta 462: 59-72.

TEDESCO MJ et al. 1995. Análise de solo, plantas e outros materiais. 2.ed. Porto Alegre: UFRGS. 174p.

THOMASI SS et al. 2015. Sequential extraction of copper, nickel, zinc, lead and cadmium from Brazilian Oxysols: metal leaching and metal distribution in soil fractions. International Journal of Environmental Studies 72: 41-55.

VANĚK A et al. 2005. Mobility of lead, zinc and cadmium in alluvial soils heavily polluted by smelting industry. Plant, Soil and Environment 51: 316-321.

VIOLANTE A et al. 2010. Mobility and bioavailability of heavy metals and metalloids in soil environments. Journal of Soil Science and Plant Nutrition 10: 268-292. 